(C) 2017 IEEE. Personal use of this material is permitted. Permission from IEEE must be obtained for all other uses, in any current or future media, including reprinting/republishing this material for advertising or promotional purposes, creating new collective works, for resale or redistribution to servers or lists, or reuse of any copyrighted component of this work in other works. 


\section{Cavity-backed proximity-coupled reconfigurable microstrip antenna with agile polarizations and steerable beams}

\author{
Shu-Lin Chen, Pei-Yuan Qin, Can Ding, and Y. Jay Guo
}

\begin{abstract}
A major challenge for a combined reconfigurable antenna is to realize both polarization switching and beam steering independently in a compact antenna structure. A cavitybacked proximity-coupled reconfigurable microstrip antenna proposed in this communication provides an efficient solution. Beam lead PIN diodes DSM8100-000 are employed as switching elements to achieve the reconfiguration. Three different linear polarizations $\left(0^{\circ}, 45^{\circ}, 90^{\circ}\right)$ are realized by switching the diodes on a proximity-coupled feed network. For each polarization state, the main beam can be steered to three directions by using a reconfigurable parasitic-element network. The parasiticelement network is printed on the same plane of the radiating patch, thereby making the antenna compact. This antenna has 9 different working modes and for all the working modes, the reflection coefficients are below $-10 \mathrm{~dB}$ with the measured realized gains ranging from 7.2-8.1 $\mathrm{dBi}$.
\end{abstract}

Index Terms-Reconfigurable antennas, polarization and pattern reconfigurable antennas, proximity-coupled antennas, beam steering, microstrip antennas.

\section{INTRODUCTION}

With rapid development of wireless communications, there has been a strong interest in reconfigurable antennas due to their capabilities of providing multiple functionalities [1]. Typical reconfigurable parameters of an antenna are frequency, polarization, radiation pattern, or a combination of the above [2][9]. For combined two-characteristic reconfigurable antennas, frequency-polarization, frequency-pattern, and polarizationpattern reconfigurable antennas have been realized [10]-[11]. Moreover, a three-characteristic reconfigurable antenna was achieved using a reconfigurable parasitic pixel surface [12]. Compared to single-parameter reconfigurable antennas, combined ones have the capacity to deliver more flexibility and diversity, which can bring significant benefits to many wireless communication systems.

Among all two-parameter reconfigurable antennas, it is the polarization-pattern that has gained substantial attention due to their strong capabilities to enhance the performance of multi-input multi-output (MIMO) systems [13]-[14]. In [14], by employing a polarization-pattern reconfigurable circular patch antenna, an average capacity improvement of $17.5 \%$ is accomplished. For this purpose, some innovative combined polarization and pattern reconfigurable antennas have been proposed [15]-[18]. In [15], combined reconfigurable antennas were proposed that can switch their operating modes between a monopole mode and a fundamental patch mode. By exciting the monopole mode, a conical pattern with a vertical polarization was obtained. For the patch mode, the antenna

This work was supported by Australia Research Council (ARC) Discovery Program under Grant DE170101203 and Grant DP160102219.

Shu-Lin Chen, Pei-Yuan Qin, Can Ding and Y. Jay Guo are with the Global Big Data Technologies Centre, University of Technology Sydney (UTS), NSW 2007, Australia (e-mail: ShulinChen.UTS@gmail.com) can achieve broadside patterns with dual orthogonal horizontal polarizations. In [16], a reconfigurable microstrip antenna was presented that can switch between a monopole mode with a vertical-linear-polarized conical pattern and a patch mode with a horizontal-circular-polarized broadside pattern. In [17], by switching between two orthogonal truncated monopoles, two orthogonal linear polarizations with omnidirectional patterns in different planes can be reconfigured. It should be noted that in the above reported designs, the pattern and the polarization cannot be independently reconfigured. In other words, the polarization/pattern changes with the pattern/polarization. This significantly limits the diversity provided by the combined reconfigurable antennas. Furthermore, the reported designs can only achieve pattern reconfiguration between different pattern shapes, e.g. the switch between broadside and conical patterns, rather than steering the main beam within a certain angle range. However, as beamforming technology is widely adopted in many wireless systems, such as $5 \mathrm{G}$ systems, it is highly desired that combined reconfigurable antennas are equipped with both the polarization agility and the beam scanning capability. Unfortunately, to date, very few reported designs can address the challenge, that is to achieve a beam scanning function with independent polarization reconfiguration in a compact antenna structure. A good solution has been provided in [18] using a microstrip patch antenna with parasitic dipoles. It can change between two orthogonal linear polarizations and one diagonal linear polarization. For each polarization, the main beam can be steered among $-45^{\circ}, 45^{\circ}$, and a dualdirection beam with realized gains of $2.5-3.5 \mathrm{dBi}$ at 2.45 GHz. For the antenna presented in [18], the parasitic elements and the radiating patch are printed in a vertical plane and a horizontal plane, respectively. Furthermore, the antenna cannot steer its beam towards broadside. A broadside radiation is usually needed in wireless communication systems.

In this communication, a planar cavity-backed microstrip antenna with three linear polarization states and beam-steering capabilities is proposed in order to significantly enhance the technology for combined reconfigurable antennas. Compared to [18], substantial advancements have been made. First, the antenna can switch between three linear polarizations and for each polarization state, the main beam in $\mathrm{H}$ plane can be steered among $\theta \approx 20^{\circ}, 0^{\circ},-20^{\circ}$ with $\theta \approx 0^{\circ}$ as the broadside direction. This combined reconfiguration is realized by employing a combined novel reconfigurable proximity-coupled feed network and a parasitic-element network. Second, the proposed antenna is a two-layer planar microstrip structure by printing the parasitic-element network on the same plane of the radiating patch. Third, in order to satisfy the increasing demand for high data rates in wireless communications, the proposed reconfigurable antenna is designed to work at 11 $\mathrm{GHz}$ with a realized gain from 7.2-8.1 $\mathrm{dBi}$.

\section{RECONFIGURABLE ANTENNA DESIGN AND ANALYSIS}

\section{A. Antenna configuration}

The geometry of the proposed cavity-backed reconfigurable microstrip antenna is shown in Fig. 1. This antenna consists of an upper substrate, a lower substrate and a cavity-backed 


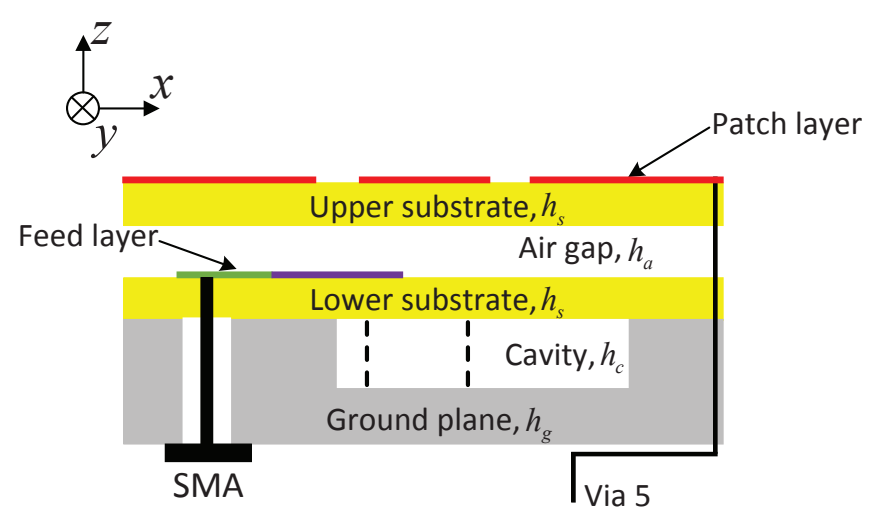

Fig. 1. Configuration of the proposed cavity-backed reconfigurable microstrip antenna.

ground plane. An air gap with a height $h_{a}$ is introduced between the upper and lower substrates. Both of these two substrates are Rogers RT/Duriod 5880 laminate $\left(\epsilon_{r}=2.2, \tan \delta=\right.$ 0.009 ) with the dimensions of $L_{s} \times L_{s} \times h_{s}$.

For the upper substrate, a metal patch layer is etched on the top, whilst the bottom metal is removed. Fig. 2 shows the top view of the patch layer. The patch layer consists of a centrallylocated square radiating patch with dimensions of $L_{p} \times L_{p}$ and a reconfigurable parasitic-element network. The network is composed of three groups (A, B and C) of parasitic lines and thin biasing strips. As can be seen, the whole structure of the patch layer is symmetrical with regards to diagonal lines $\mathrm{PP}^{\prime}$ and $\mathrm{QQ}^{\prime}$. The three groups of parasitic lines are placed around the radiating patch to achieve pattern reconfiguration. The parasitic line group A and group B have the lengths of $L_{1}$ and $L_{2}$, respectively. Both of them have a distance of $d_{\mathrm{H}}$ to the radiating patch. The parasitic line group $\mathrm{C}$ has a length of $L_{3}$ with a distance of $d_{\mathrm{V}}$ to the radiating patch. All these parasitic lines have a width of $W$. Four diodes D1 $\sim$ D4 are soldered between group A and group B, and another four diodes D5 $\sim$ D8 are soldered between group B and group C. The DC biasing powers are applied through eight metallic vias ( Via $1 \sim$ Via 8 ) and thin strips. All the eight metallic vias are drilled from the upper substrate to the bottom ground plane and the DC biasing lines are located behind the ground plane to reduce their effects on the antenna performance. In order to choke RF signals while maintaining the DC continuity, the thin strips are broken down into small sections and gaps between the sections are bridged with inductors. Here, only the detailed structure of Via 5 with its biasing line is shown in Fig. 1 for simplicity.

For the lower substrate, a metal feed layer is printed on the top, whilst the bottom metal is removed. Fig. 3 shows the details of the top view of the feed layer. A diagonal feed line with a width of $W_{d}$ is connected to an SMA feed pin and a thin slot is cut for soldering a capacitor to choke the DC signal while maintaining the RF continuity. Two diodes D9 and D10 are soldered between the diagonal feed line and two symmetrical branch feed lines to achieve the polarization reconfiguration. Both of the two branch feed lines have a length of $L_{a}+L_{b}$ and a width of $W_{f}$. The DC biasing voltages are applied through three metallic vias (Via $9 \sim$ Via 11) and

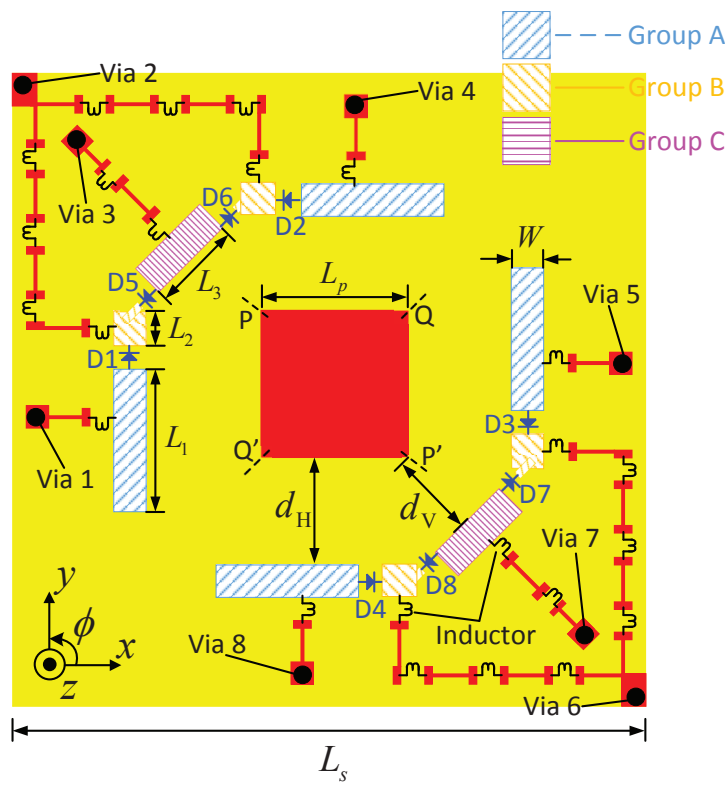

Fig. 2. Top view of the patch layer.

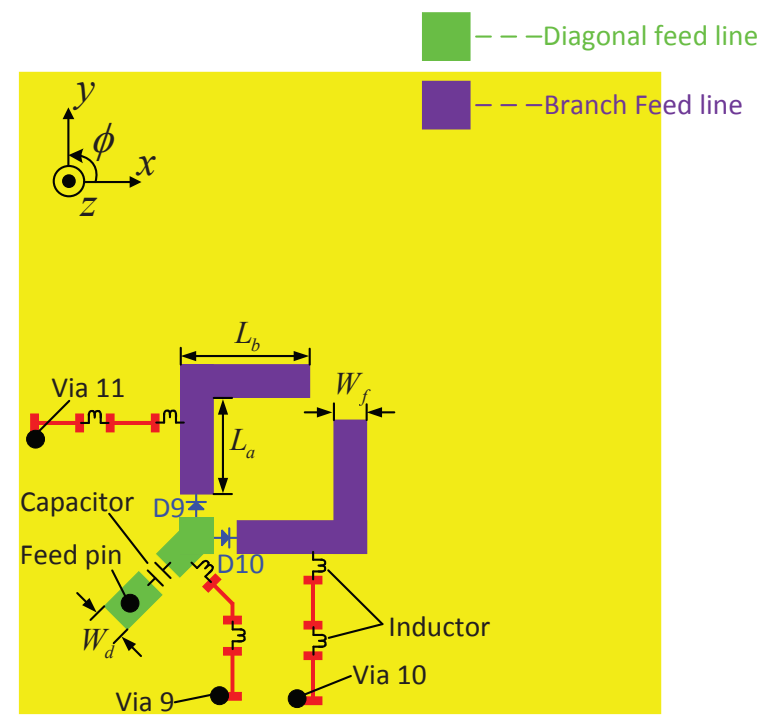

Fig. 3. Top view of the feed layer.

thin strips to control the diodes D9 and D10. Under the lower substrate, it is an L-shape cavity-backed ground plane with a thickness of $h_{g}$. Top view of the cavity-backed ground plane is shown in Fig. 4, and the dimensions of the air cavity are $L_{c} \times W_{c} \times h_{c}$. This cavity-backed ground plane is employed for the impedance match [19]-[20].

\section{B. Polarization reconfiguration and beam steering mechanism}

The proposed antenna is able to reconfigure the polarization states and steer its main beam independently. As can be seen, the antenna uses a proximity-coupled feed network with an air gap introduced between the feed lines and the radiating patch. Thus, polarization reconfiguration can be realized by reconfiguring the feed lines. Meanwhile, the air gap provides enough space to place the reconfigurable feed network on 


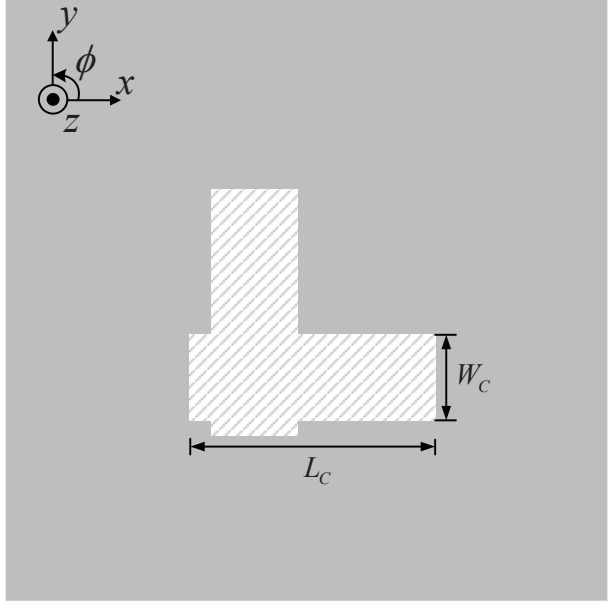

Fig. 4. Top view of the cavity-backed ground plane.

TABLE I

NINE RECONFIGURABLE WORKING MODES

\begin{tabular}{c|c|c|c}
\hline \multirow{2}{*}{ Working mode } & Diodes switched ON & Polarization & Pattern mode \\
\hline Mode 1 & D9 & $0^{\circ}$ & Broadside \\
\hline Mode 2 & D9 D2 & $0^{\circ}$ & Left $(-y)$ \\
\hline Mode 3 & D9 D4 & $0^{\circ}$ & Right $(+y)$ \\
\hline Mode 4 & D10 & $90^{\circ}$ & Broadside \\
\hline Mode 5 & D10 D3 & $90^{\circ}$ & Left $(-x)$ \\
\hline Mode 6 & D10 D1 & $90^{\circ}$ & Right $(+x)$ \\
\hline Mode 7 & D9 D10 & $45^{\circ}$ & Broadside \\
\hline Mode 8 & D9 D10 D7 D8 & $45^{\circ}$ & Left $(-x,+y)$ \\
\hline Mode 9 & D9 D10 D5 D6 & $45^{\circ}$ & $\operatorname{Right}(+x,-y)$ \\
\hline \hline
\end{tabular}

the feed layer. Once the polarization state is selected, the beam steering can be conducted by switching diodes D1 D8 between the three-group parasitic lines.

For the polarization reconfiguration, as shown in Fig. 3, two PIN diodes D9 and D10 are used to connect or disconnect the diagonal feed line and two branch feed lines. Three different polarizations can be reconfigured by controlling the states of these two diodes. When D9 is ON and D10 is OFF, $\phi=0^{\circ}$ polarization which is aligned with $x$ axis can be obtained. Similarly, $\phi=90^{\circ}$ polarization along $y$ axis can be obtained when D9 is OFF and D10 is ON. When both D9 and D10 are $\mathrm{ON}, \phi=45^{\circ}$ polarization along the diagonal line can be obtained by equally combining the $x$-axis current and $y$-axis current.

For each of these three polarization states, beam steering can be obtained by using the reconfigurable parasitic-element network as shown in Fig. 2. The basic mechanism of the beam steering is to consider the radiating patch with two parasitic lines as a three-element array. The beam can be steered by changing the phase of the parasitic lines. Take the $y$-polarized $\left(\phi=90^{\circ}\right)$ state as an example as shown in Fig. 5(a), the center radiating patch has a current $\overrightarrow{\mathrm{I}}_{1}=\mathrm{I}_{1} \mathrm{e}^{j \psi_{1}} \vec{y}$, where $\vec{y}$ represents unit current vector along $y$ axis. Two different parasitic lines have coupled currents $\overrightarrow{\mathrm{I}}_{2}=\mathrm{I}_{2} e^{j \psi_{2}} \vec{y}$ and $\overrightarrow{\mathrm{I}}_{3}=\mathrm{I}_{3} e^{j \psi_{3}} \vec{y}$. The phases of coupled currents are determined by lengths and positions of the parasitic lines. In this work, the positions of the parasitic lines are fixed and only their lengths are changeable.
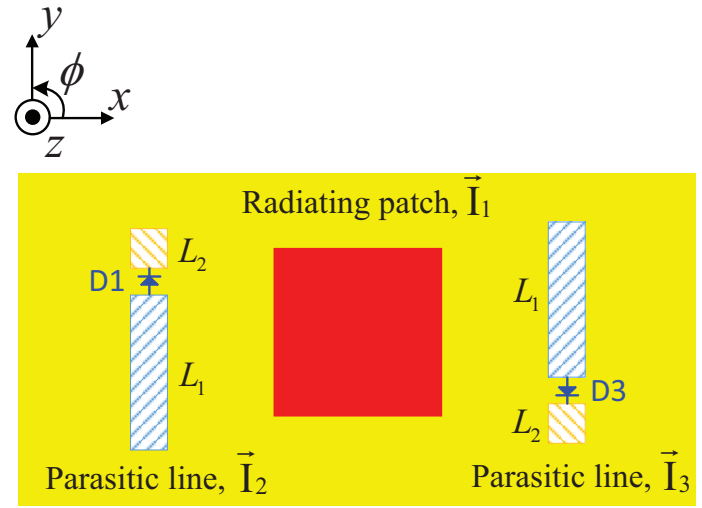

(a)
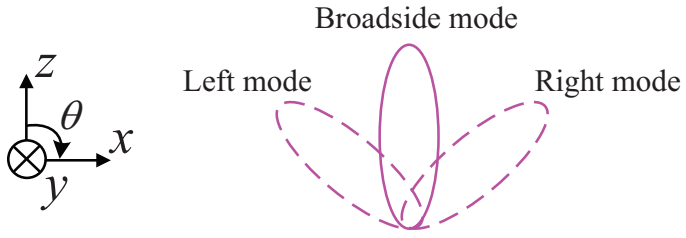

(b)

Fig. 5. Demonstration of beam steering mechanism. (a) Three-element array, and (b) pattern modes.

More specifically, when both the diodes D1 and D3 are OFF, the two active parasitic lines are of the same lengths $\left(L_{1}\right)$. In this case, the main beam is towards the broadside direction which is defined as Broadside mode, as shown in Fig. 5(b). When D1 is ON and D3 is OFF, the left active parasitic line $\left(L_{1}+L_{2}\right)$ is longer than the right one $\left(L_{1}\right)$. In this case, the main beam steers to $+x$ axis direction, which is defined as Right mode. On the contrary, when D1 is OFF and D3 is ON, the pattern is steered to $-x$ axis direction, which is defined as Left mode.

Based on this working mechanism, the pattern reconfiguration for $\phi=0^{\circ}$ polarization state can be achieved using the parasitic lines group A and group B with the switching diodes D2 and D4, as can be seen in Fig. 2. Parasitic lines group B and group $\mathrm{C}$ with diodes D5 $\sim$ D8 are utilized to switch the beam for the $\phi=45^{\circ}$ polarization state. Thus, for each of these three polarization states, three modes that are Broadside mode, Left mode, and Right mode can be achieved by controlling the diodes. Overall, the antenna has nine working modes. Table I shows the working states of the PIN diodes for each mode.

\section{DC feed network design}

The proposed antenna operates at $11 \mathrm{GHz}$. Usually, for relative low working frequencies such as $2.4 \mathrm{GHz}$, the DC lines are very thin in terms of the wavelength. Thus, the DC biasing lines can be treated as high impedance lines, and few currents will be coupled on the feed lines. However, at $11 \mathrm{GHz}$, DC lines could have much more effects on the antenna performance as the electric sizes of the DC lines have largely increased. Therefore, the DC feed network should be carefully optimized. The biasing network in this work consists 
TABLE II

OPTIMIZED PARAMETERS FOR CAVITY-BACKED PROXIMITY-COUPLED RECONFIGURABLE MICROSTRIP ANTENNA

\begin{tabular}{|c|c|c|c|c|c|c|c|c|}
\hline Parameter & $h_{s}$ & $h_{a}$ & $L_{s}$ & $h_{g}$ & $L_{p}$ & $W_{f}$ & $L_{c}$ & $W_{c}$ \\
\hline Value $(\mathrm{mm})$ & 0.508 & 2 & 32 & 4 & 6.5 & 1.5 & 12.5 & 5 \\
\hline Parameter & $h_{c}$ & $L_{1}$ & $L_{2}$ & $L_{3}$ & $L_{a}$ & $L_{b}$ & $d_{\mathrm{H}}$ & $d_{\mathrm{V}}$ \\
\hline Value $(\mathrm{mm})$ & 3 & 7.1 & 2.25 & 5.6 & 6.2 & 7 & 5 & 3.8 \\
\hline
\end{tabular}

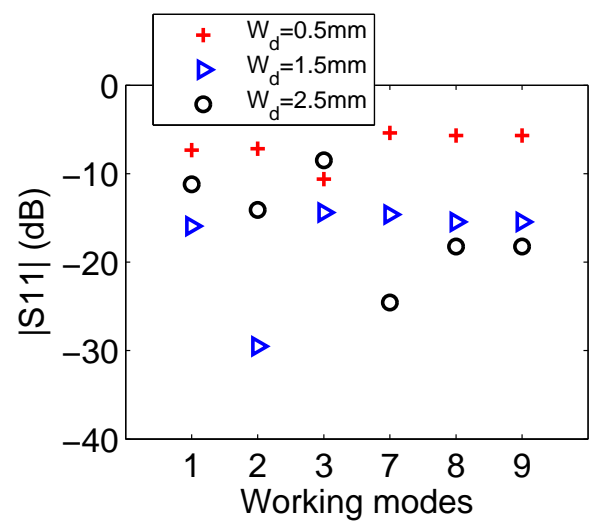

Fig. 6. Input reflection coefficients versus feed line width of $W_{d}$ for different working modes.

of metallic feed vias and thin strips. In order to reduce the power coupled on the feed vias ( Via $1 \sim$ Via 11 ), the positions of the vias have been carefully chosen where little effects have been found on the antenna's S-parameters and radiation patterns. Furthermore, in order to reduce the effects of the biasing strip lines on the antenna, the strip lines are broken down into small sections and the gaps between the sections are bridged with inductors, as shown in Figs. 2 and 3.

Diodes used in this design are beam lead PIN diodes DSM8100-000 [21], which can work up to $25 \mathrm{GHz}$. According to the datasheet, the diode can be modeled as a resistance of $4 \Omega$ for the ON state and as a parallel circuit with a capacitance of $0.03 \mathrm{pF}$ and a resistance of $20 \mathrm{k} \Omega$ for the OFF state. Ten diodes will be used as the switching elements. All the surface-mount inductors are Coilcraft chip inductors 0402HP-3N6X_LU $(3.6 \mathrm{nH})$ with a self-resonant frequency at $11.7 \mathrm{GHz}$. They can choke the RF signal while maintaining DC signals at $11 \mathrm{GHz}$. In order to avoid the DC signal flowing into the coaxial feed, a capacitor with $2 \mathrm{pF}$ is used on the diagonal feed line, as shown in Fig. 3.

\section{Parameters STUdy AND Discussion}

The antenna is studied and simulated using full-wave simulation tool ANSYS HFSS 2016 [22]. The final parameters of the antenna are given in Table II. For the proposed polarization and pattern reconfigurable antenna, one challenge is to realize acceptable impedance matches for the three polarization states. The input impedances of the $0^{\circ}$ and $90^{\circ}$ polarization states are the same, while they are different from that of the $45^{\circ}$ polarization state. In order to achieve acceptable reflection coefficients for all these three states, the diagonal feed line width $W_{d}$ is optimized. A parametric study of $W_{d}$ was conducted and the results are shown in Fig. 6. As can be

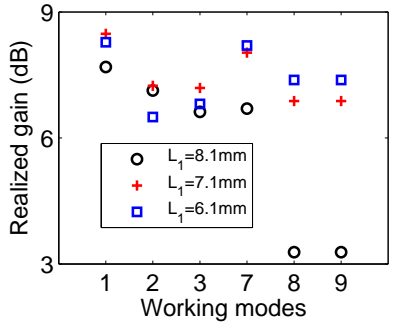

(a)

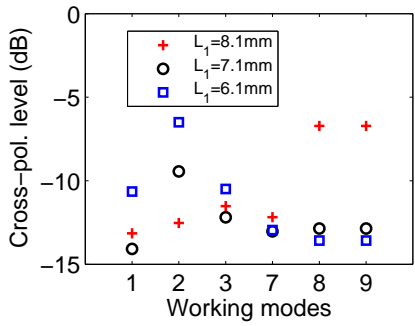

(b)
Fig. 7. Realized gains and cross-polarization levels versus parasitic line length of $L_{1}$ for different working modes. (a) Realized gains, and (b) crosspolarization levels.

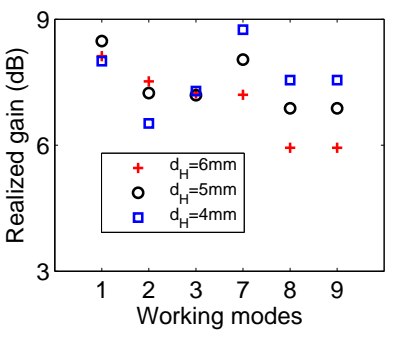

(a)

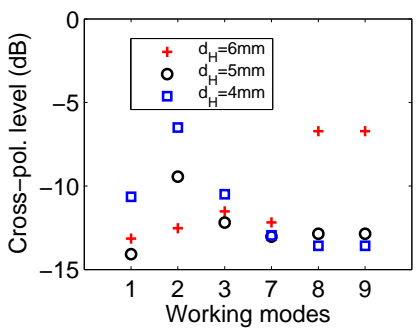

(b)
Fig. 8. Realized gains and cross-polarization levels versus distance of $d_{\mathrm{H}}$ for different working modes. (a) Realized gains, and (b) cross-polarization levels.

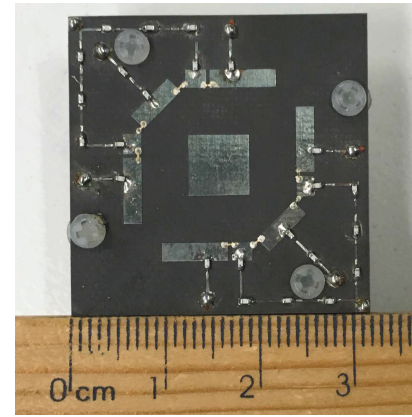

(a)

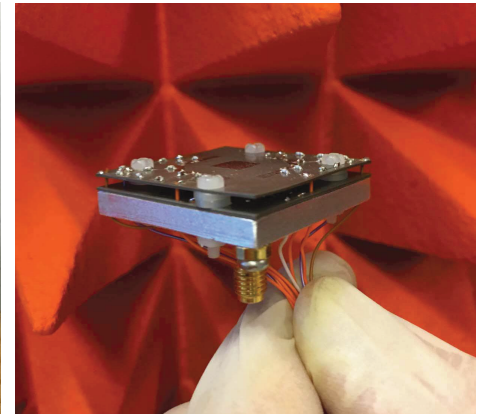

(b)
Fig. 9. Prototype of the proposed cavity-backed reconfigurable microstrip antenna. (a)Top view, and (b) overall view.

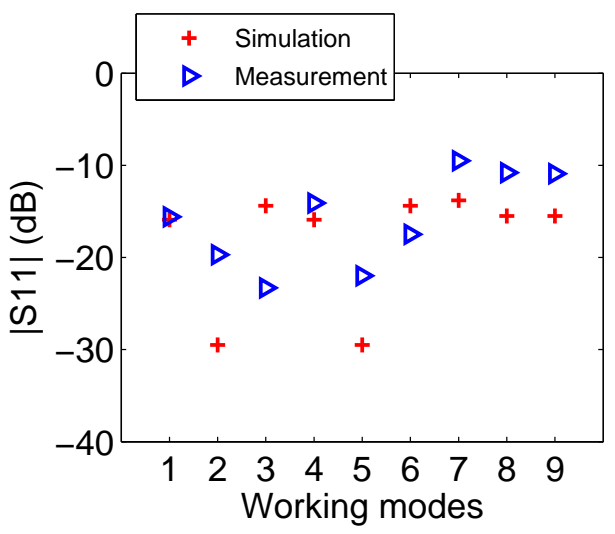

Fig. 10. Simulated and measured $|\mathrm{S} 11|$ at $11 \mathrm{GHz}$ for all the nine working modes 


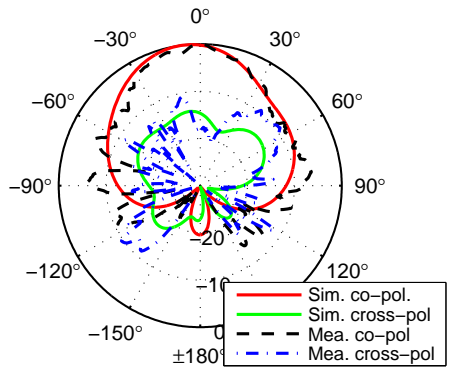

(a)

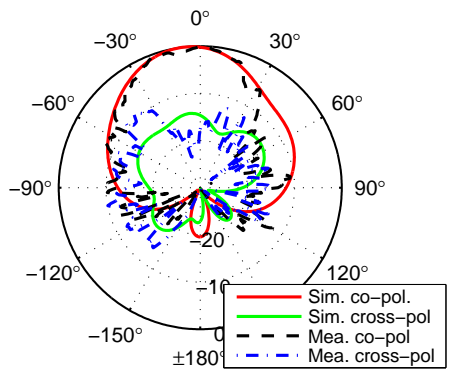

(d)

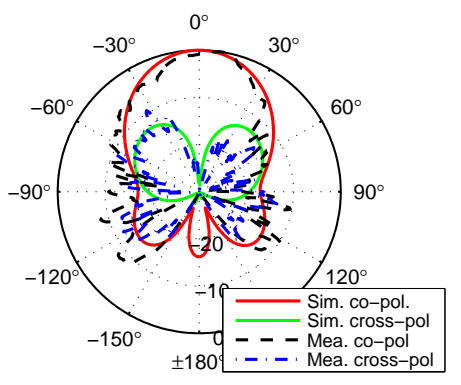

(g)

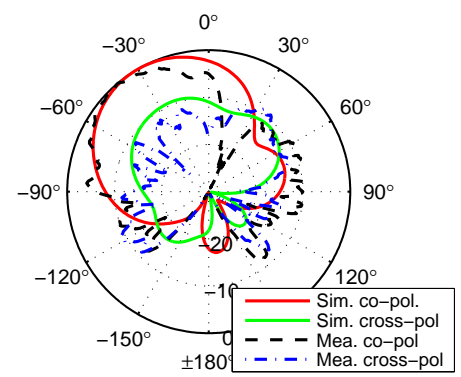

(b)

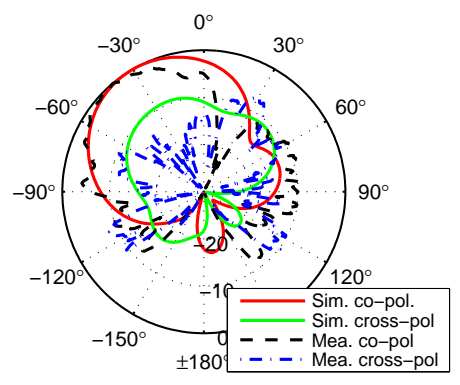

(e)

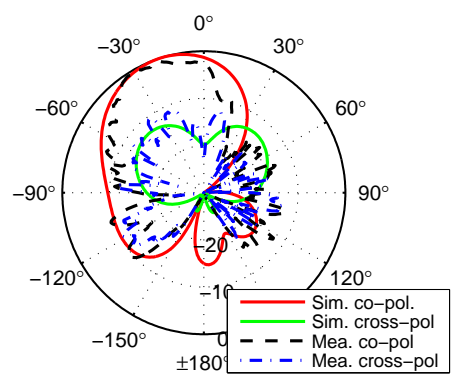

(h)

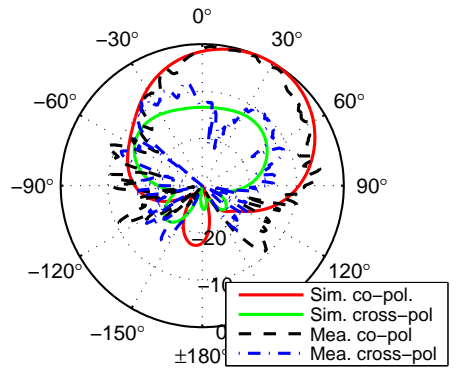

(c)

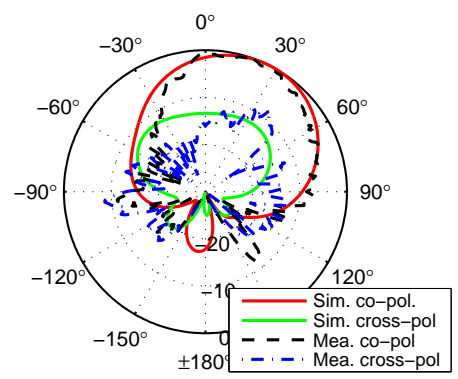

(f)

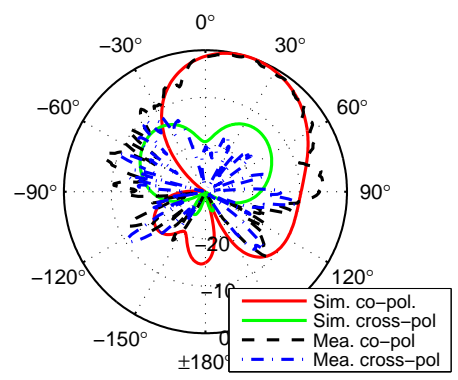

(i)

Fig. 11. Simulated and measured H-plane radiation patterns at $11 \mathrm{GHz}$ for all the nine working modes. (a) Mode 1, (b) Mode 2, (c) Mode 3, (d) Mode 4, (e) Mode 5, (f) Mode 6, (g) Mode 7, (h) Mode 8, and (i) Mode 9.

seen, when $W_{d}=0.5 \mathrm{~mm}$, the antenna has a high reflection for all working modes. For $W_{d}=2.5 \mathrm{~mm}$, the antenna has a relative high reflection at working Mode 3 . Thus, to achieve an acceptable $|\mathrm{S} 11|$ for all the working modes, $W_{d}=1.5 \mathrm{~mm}$ is selected.

As discussed in Section II, the coupling between the radiating patch and the parasitic lines is crucial for the pattern reconfiguration. The lengths of parasitic lines affect radiation patterns. Furthermore, the distance between radiating patch and parasitic lines plays an important role in titling the pattern. Here, parameter studies on the length of $L_{1}$ and distance of $d_{\mathrm{H}}$ are shown in Fig. 7 and Fig. 8, respectively. Fig. 7 shows the realized gains and cross-polarization levels versus the length $L_{1}$ for each working mode. As can be seen from Fig. 7, when $L_{1}=8.1 \mathrm{~mm}$, the realized gains reduce to about $3 \mathrm{dBi}$ for working modes 8 and 9 . When $L_{1}=7.1 \mathrm{~mm}$ and $L_{1}=6.1$ $\mathrm{mm}$, the realized gains are relatively more stable for all the working modes. In addition, the cross-polarization levels for $L_{1}=6.1 \mathrm{~mm}$ are higher than the ones for $L_{1}=7.1 \mathrm{~mm}$. Therefore, $L_{1}=7.1 \mathrm{~mm}$ is selected. Fig. 8 shows the realized gains and cross-polarization levels versus the length $d_{H}$ for each working mode. When $d_{H}=5 \mathrm{~mm}$, the antenna can achieve more stable gains than the ones with $d_{H}=6 \mathrm{~mm}$ and $d_{H}=4 \mathrm{~mm}$. Moreover, when $d_{H}=5 \mathrm{~mm}$, the crosspolarization levels for all working modes are relative lower. Therefore, $d_{H}=5 \mathrm{~mm}$ is selected.

\section{RESULTS AND DISCUSSION}

Based on the above analysis, an antenna prototype was fabricated as shown in Fig. 9. The PIN diodes are attached to the antenna using electrically conductive silver epoxy (EPO-TEK H70E-1LB Kit from Epoxy Technology). A Keithley 2230 SourceMeter power supply is employed to provide constant currents for biasing the diodes. According to the datasheet of PIN diode DSM8100-000, a typical power to turn on one diode is a $10 \mathrm{~mA}$ current with a voltage of $0.95 \mathrm{~V}$ [21]. For all the nine working modes of the proposed polarization and pattern reconfigurable antenna, the power consumption is in the range of $0.095 \mathrm{~W}$ (One PIN diode is ON) to $0.38 \mathrm{~W}$ (Four diodes are $\mathrm{ON}$ ). By switching the ten diodes, nine working modes with different radiation performance can be achieved. Fig. 10 shows the simulated and measured S-parameters at 
TABLE III

SIMULATED AND MEASURED REALIZED GAINS FOR ALL THE NINE WORKING MODES

\begin{tabular}{c|c|c|c|c|c|c|c|c|c}
\hline \multirow{2}{*}{ Realized gain } & \multicolumn{3}{|c|}{$0^{\circ}$ polarization } & \multicolumn{3}{c|}{$90^{\circ}$ polarization } & \multicolumn{3}{c}{$45^{\circ}$ polarization } \\
\cline { 2 - 11 } & Mode 1 & Mode 2 & Mode 3 & Mode 4 & Mode 5 & Mode 6 & Mode 7 & Mode 8 & Mode 9 \\
\hline Simulation (dBi) & 8.48 & 7.24 & 7.19 & 8.48 & 7.24 & 7.19 & 8.04 & 6.88 & 6.88 \\
\hline Measurement (dBi) & 8.04 & 7.22 & 7.38 & 7.83 & 7.62 & 7.81 & 8.12 & 7.42 & 7.25 \\
\hline \hline
\end{tabular}

$11 \mathrm{GHz}$ for each mode. As can be seen, the measured Sparameters agree reasonably well with the simulated ones, and the reflection coefficients are all below $-10 \mathrm{~dB}$ at 11 $\mathrm{GHz}$. Some discrepancies can be found, which could be mostly attributed to the individual differences of the PIN didoes. Furthermore, the inaccuracies of the fabrication process, such as the biasing network, may affect the antenna performance.

Far-field radiation patterns were measured for different modes of the proposed antenna using a spherical near-field (SNF) antenna measurement system NSI-700S-50 located at CSIRO, Australia. For H-plane radiation patterns, as can be seen from Fig. 11, the measured results agree reasonably well with the simulated ones. Table III shows the simulated and measured realized gains at all the 9 working modes. At $\phi=0^{\circ}$ polarization state, the measured gains can achieve $8.04 \mathrm{dBi}$, $7.22 \mathrm{dBi}$, and $7.38 \mathrm{dBi}$ for Mode $1 \sim$ Mode 3. Moreover, the measured half-power beamwidth (HPBW) can cover from $-24^{\circ} \sim 21^{\circ},-68^{\circ} \sim-17^{\circ}$, and $-7^{\circ} \sim 48^{\circ}$, respectively. It means that the HPBW for $0^{\circ}$ polarization can reach a wide angle from $-68^{\circ} \sim 48^{\circ}$ with a gain around $7.2 \mathrm{dBi}$. Similarly, at $\phi=90^{\circ}$ polarization state, for Mode $4 \sim$ Mode 6 , the measured gains can reach $7.83 \mathrm{dBi}, 7.62 \mathrm{dBi}$, and 7.81 dBi. Meanwhile, the HPBW can cover from $-68^{\circ} \sim 50^{\circ}$. At $\phi=45^{\circ}$ polarization state, the measured gains for the three modes are $8.12 \mathrm{dBi}, 7.42 \mathrm{dBi}$, and $7.25 \mathrm{dBi}$ respectively. The HPBW patterns can cover from $-49^{\circ} \sim 49^{\circ}$.

\section{CONCLUSION}

In this communication, the technology for combined pattern and polarization reconfigurable antennas has been advanced by proposing a novel cavity-backed proximity-coupled reconfigurable microstrip antenna. It can switch between three linear polarization states $\phi=0^{\circ}, \phi=45^{\circ}, \phi=90^{\circ}$. For each polarization state, the main beam in $\mathrm{H}$ plane can be steered to about $20^{\circ}, 0^{\circ},-20^{\circ}$ with a relative stable gain performance by reconfiguring the feed network and the parasitic-element network. For all these working modes, the $|\mathrm{S} 11|$ are below $-10 \mathrm{~dB}$ with the realized gains ranging from 7.2-8.1 $\mathrm{dBi}$. The pattern and polarization diversities provided by the antenna can be exploited to improve the performance of various wireless systems including MIMO systems, cognitive radio systems, and multifunction consumer wireless devices. Future work will focus on introducing frequency agility to the antenna to realize a fully reconfigurable antenna.

\section{REFERENCES}

[1] C. G. Christodoulou, Y. Tawk, S. A. Lane, and S. R. Erwin, "Reconfigurable antennas for wireless and space applications," in Proc. IEEE, vol. 100, no. 7, pp. 2250-2261, Jul. 2012.

[2] S. Gao, A. Sambell, and S. S. Zhong, "Polarization-agile antennas," IEEE Antennas Propag. Mag., vol. 48, no. 3, pp. 28-37, Jun. 2006.
[3] S. Genovesi, A. D. Candia, and A. Monorchio, "Compact and low profile frequency agile antenna for multistandard wireless communication systems," IEEE Trans. Antennas Propag., vol. 62, no. 4, pp. 1019-1026, Mar. 2014.

[4] Y. Li, Z. Zhang, W. Chen, and Z. Feng, "Polarization reconfigurable slot antenna with a novel compact CPW-to-slotline transition for WLAN application," IEEE Antennas Wireless Propag. Lett., vol. 9, pp. 252-255, 2010.

[5] W. Lin and H. Wong, "Polarization reconfigurable wheel-shaped antenna with conical-beam radiation pattern," IEEE Trans. Antennas Propag., vol. 63, no. 2, pp. 491-499, Feb. 2015.

[6] W. Lin and H. Wong, "Wideband circular polarization reconfigurable antenna," IEEE Trans. Antennas Propag., vol. 63, no. 12, pp. 5938-5944, Dec. 2015.

[7] S. Zhang, G. H. Huff, J. Feng, and J. T. Bernhard, "A pattern reconfigurable microstrip parasitic array," IEEE Trans. Antennas Propag., vol. 52, no. 10 , pp. $2773-2776$, Oct. 2004

[8] J.-S Row and C.-W. Tsai, "Pattern reconfigurable antenna array with circular polarization," IEEE Trans. Antennas Propag., vol. 64, no. 4, pp. 1525-1530, Apr. 2016.

[9] L.-Y. Ji, Y. J. Guo, P.-Y. Qin, S. X. Gong, and R. Mittra, "A reconfigurable partially reflective surface (PRS) antenna for beam steering," IEEE Trans. Antennas Propag., vol. 63, no. 6, pp. 2387-2395, Jun. 2015.

[10] N. Nguyen-Trong, L. Hall, and C. Fumeaux, "A frequency- and polarization-reconfigurable stub-Loaded microstrip patch antenna," IEEE Trans. Antennas Propag., vol. 63, no. 11, pp. 5235-5240, Oct. 2015.

[11] N. Nguyen-Trong, L. Hall, and C. Fumeaux, "A frequency- and patternreconfigurable center-shorted microstrip antenna," IEEE Antennas Wireless Propag. Lett., vol. 15, pp. 1955-1958, Mar. 2016.

[12] D. Rodrigo, B. A. Cetiner, and L. Jofre, "Frequency, radiation pattern and polarization reconfigurable antenna using a parasitic pixel layer," IEEE Trans. Antennas Propag., vol. 62, no. 6, pp. 3422-3427, Jun. 2014.

[13] A. Grau, H. Jafarkhani, and F. De Flaviis, "A reconfigurable multipleinput multiple-output communication system," IEEE Trans. Wireless Commun., vol. 7, no. 5, pp. 1719-1733, May 2008

[14] D. Piazza, P. Mookiah, M. D'Amico, and K. R. Dandekar, "Experimental analysis of pattern and polarization reconfigurable circular patch antennas for MIMO systems," IEEE Trans. Antennas Propag., vol. 62, no. 6, pp. 3422-3427, Jun. 2014.

[15] W.-L. Liu, T.-R. Chen, S.-H. Chen, and J.-S. Row, "Reconfigurable microstrip antenna with pattern and polarisation diversities," Electron. Lett., vol. 43, no. 2, pp. 77-78, 2007.

[16] W. Cao, B. Zhang, A. Liu, T. Yu, D. Guo, and K. Pan, "A reconfigurable microstrip antenna with radiation pattern selectivity and polarization diversity," IEEE Antennas Wireless Propag. Lett., vol. 11, pp. 453-456, 2012.

[17] S. Raman, P. Mohanan, N. Timmons, and J. Morrison, "Microstrip-fed pattern- and polarization- reconfigurable compact truncated monopole antenna," IEEE Antennas Wireless Propag. Lett., vol. 12, pp. 710-713, 2013.

[18] C. Gu et al., "Compact smart antenna with electronic beam-switching and reconfigurable polarizations," IEEE Trans. Antennas Propag., vol. 63, no. 12, pp. 5325-5333, Dec. 2015.

[19] D. Sun and L. You, "A broadband impedance matching method for proximity-coupled microstrip antenna," IEEE Trans. Antennas Propag., vol. 58 , no. 4, pp. 1392-1397, Apr. 2010

[20] Y. Liu, S.-L. Chen, Y. Ren, J. Cheng and Q. H. Liu, "A broadband proximity-coupled dual-polarized microstrip antenna with L-shape backed cavity for X-band applications," Int. J. Electron. Commun., vol. 69, no. 9, pp. 1226-1232, 2015.

[21] Skyworks Solutions Inc. "DSM8100-000: Mesa BeamLead PIN Diode," Aug. 2008. [Online]. Available: http://www.skyworksinc.com/Product/251/DSM8100-000.

[22] ANSYS HFSS-High Frequency Electromagnetic Field Simulation. [Online]. Available: http://www.ansys.com/Products/Electronics/ANSYSHFSS. 